\title{
Measuring global solar irradiance and atmospheric optical thickness, using a digital pyranometer under conditions of clear skies and no clouds in Valencia (Venezuela)
}

\section{Medición de la irradiancia solar global y del espesor óptico atmosférico, mediante un piranómetro digital, en condiciones de cielos claros y sin nubes, en Valencia (Venezuela)}

\author{
Nelson Falcón( $\left.{ }^{*}\right)$, Jesús Palacios \\ Laboratorio de Física de la Atmosfera y el Espacio Ultraterrestre, Departamento de Física, Facultad Experimental de \\ Ciencias y Tecnología, Universidad de Carabobo, Venezuela. \\ (ग) Email: nelsonfalconv@gmail.com \\ Received / Recibido: 13/04/2013. Revised / Revisado: 08/06/2013. Accepted / Aceptado: 24/06/2013.
}

DOI: http://dx.doi.org/10.7149/OPA.46.3.295

\begin{abstract}
:
The determination of the local solar irradiance and its annual change, well as quantification of atmospheric optical thickness (EOA) is important to assess environmental conditions and air quality in a given region, with multiple practical applications. In this paper we evaluate the performances of irradiance and calculate the atmospheric optical thickness in the city of Valencia (Venezuela). The irradiance measurements are performed by implementing a set of digital pyranometers, simply constructed using LEDs as photosensor elements, and record the solar elevation angle. The average values of irradiance for the city of Valencia (Venezuela) are of $805 \mathrm{~W} / \mathrm{m}^{2}$ for $620 \mathrm{~nm}$ and $249 \mathrm{~W} / \mathrm{m}^{2}$ at $472 \mathrm{~nm}$ with maximum irradiance values at $12: 31$ and 12:18 respectively HLV, the average EOA sample, a minimum true astronomical midday (12:02 HLV). We conclude that the local variation in irradiance is correlated with the atmospheric optical thickness and irradiance in the blue band of the spectrum is predominantly diffuse, and contribution in the red band is the combination of the direct and diffuse components.
\end{abstract}

Key words: Atmospheric Optical Thickness, Pyranometer Irradiance.

\section{RESUMEN:}

La determinación de la Irradiancia Solar local y su variacion anual, asi como la cuantificación del espesor óptico atmosférico (EOA) es importante para evaluar las condiciones ambientales y la calidad del aire de una región determinada, con multiples aplicaciones prácticas. En el presente trabajo se evalúan las características de la irradiancia y se calcula el espesor óptico atmosférico en la ciudad de Valencia (Venezuela). Las medidas de irradiancia se realizan mediante la implementación de un set de piranómetros digitales, construidos en forma simple mediante diodos LED como elementos fotosensores, y registran el ángulo de elevación Solar. Los valores promedio de irradiancia para la ciudad de Valencia (Venezuela) son de $805 \mathrm{~W} / \mathrm{m}^{2}$ para los $620 \mathrm{~nm}$ y $249 \mathrm{~W} / \mathrm{m}^{2}$ a $472 \mathrm{~nm}$ con valores máximo de irradiancia a las 12:31 y 12:18 HLV (TU-4:30) respectivamente; el EOA promedio muestra, un mínimo al mediodía astronómico verdadero (12:02 HLV). Se concluye que la variación de la irradiancia local está correlacionada con el espesor óptico atmosférico y que la irradiancia en el espectro del azul es predominantemente difusa, y la contribución en la banda roja es la combinación de las componentes directa y difusa.

Palabras clave: Espesor Óptico Atmosférico, Piranómetro, Irradiancia. 


\section{REFERENCES AND LINKS / REFERENCIAS Y ENLACES}

[1]. F. Kasten, A. T. Young, "Revised optical air mass tables and approximation formula", Appl. Opt. 28, 4735-4738 (1989). DOI

[2]. C. Gueymard, "The sun's total and spectral irradiance for solar energy applications and solar energy model", Sol. Energy 76, 423-453 (2004). DOI

[3]. N. Falcón, H. Peña, H. Mavo, R. Muñoz, "Irradiancia Solar Global en la Ciudad de Valencia”, Ingenieria UC 8 (2), 36-41 (2001).

[4]. I. Samsón, R. Echarri, S .Vera, A. Sartarelli, E. Cyrulies, "Medición de la radiación solar en Santo Domingo", Ciencia y Sociedad XXXV (4) 555-565 (2010).

[5]. J. Wrigh, "Medición y predicción de la radiación solar global ultravioleta (0.295-0.385 $\mu \mathrm{m})$ en condiciones de cielos claros y sin nubes", Uniciencia 22, 45-54 (2008).

[6]. G. A. Salazar, C. F. Raichijk, F. Farfan, "Estimación del coeficiente de turbidez b de ångström a partir de mediciones de irradiancia global y directa. Caso de estudio: Ciudad de Salta (Argentina)", Avances en Energías Renovables y Medio Ambiente 14 (11), 57-61 (2010).

[7]. J. C. Antuña, C. Hernández, R. Estevan, B. Barja, A. Fonte, T.Hernández, J. C. Antuña Jr., "La serie rescatada de observaciones de radiación solar de Camagüey: Aplicaciones preliminares”, Opt. Pura Apl. 44, 43-48 (2011).

[8]. M. I. Micheletti, E. A. Luccini, R. D. Piacentini, "Medicion absoluta y modelización de radiación solar espectral directa de $305.5 \mathrm{~nm}$ en Rosario, Argentina", http://www.solarlight.com/download/asades98.pdf

[9]. http://bmb.lcd.lu/science/230VAC LED

\section{Introducción}

El estudio de la irradiancia solar o cantidad de energía solar que llega a una superficie por unidad de área y por unidad de tiempo, para una localidad determinada es importante debido a sus implicaciones prácticas. Cuando el cielo está sin nubes y libre de brumas (cielos claros) la radiación solar directa que llega a la superficie es máxima y su valoración, para un lugar determinado, es empleado en los modelos de insolación. Cuantificar la máxima irradiancia, sirve por una parte, para evaluar la factibilidad y eficiencia de las tecnologías solares y las actividades agrícolas; por otra parte, puede emplearse para modelar la optimización de sistemas de refrigeración y aire acondicionado, y en la protección contra los efectos de la radiación sobre los seres vivos y la salud. También la determinación espesor óptico atmosférico (EOA), calculada a partir de la irradiancia solar, permite evaluar la presencia de aerosoles y la turbidez de la atmósfera local, que informa sobre la calidad del aire y los efectos contaminantes de los aerosoles.

La radiación solar global que incide sobre un plano horizontal, a nivel de la superficie terrestre, estará formada por la suma de una componente directa del Sol; sin desviaciones; y otra difusa emitida por las moléculas atmosféricas después del calentamiento por efecto de absorción de radiación solar, y que se difunde por todo el cielo, aparentando que es éste quien la irradia.

Si no existiera atmosfera evidentemente toda la radiación sería directa y el cielo se observaría negro, resultando la irradiancia un valor constante (constante solar extraterrestre) $I_{c s}=1367 \mathrm{~W} / \mathrm{m}^{2} \pm 1.6 \mathrm{~W} / \mathrm{m}^{2}$, y su camino óptico hasta la superficie terrestre en un lugar determinado, se verán afectada por: (1) variación estacional debido a la traslación terrestre, (2) posición del sol con respecto al meridiano (variación horaria), (3) oblicuidad del eje terrestre (declinación $\delta$ ) y (4) variaciones locales: debidas a la geografía del lugar de medición (altitud y latitud).

La variación, producto del movimiento de traslación de la tierra, de la Constante Solar Extraterrestre para un día del año en específico puede ser calculada mediante la siguiente expresión [1]:

$$
I_{0}=I_{c s}\left[1-0.0335 \sin \left(\frac{360}{365}(d-95)\right)\right] \text {, }
$$


donde $I_{0}$ es la irradiancia calculada teóricamente producto del alejamiento y acercamiento de la Tierra, debido al movimiento de translación. $I_{c s}$ es la constante solar extraterrestre y $d$ es el día del año, siendo 1 para el 01 de Enero y 365 para el 31 de Diciembre.

En un típico día despejado la radiación directa es varias veces superior a la difusa. La componente difusa puede variar desde un $20 \%$ sobre el globo en un día claro, a un $100 \%$ en un día nublado. Las nubes y la humedad atmosférica difunden la radiación solar más fuertemente que el aire seco, por lo que en un día nublado toda la radiación que recibe la superficie terrestre será difusa, los aerosoles también contribuyen a la opacidad atmosférica. El espesor óptico atmosférico $\left(\tau^{\prime}(z)\right)$ es una medida de la claridad del aire en una columna vertical de atmósfera y se calcula a partir de la ecuación de Lambert-Beer[2]:

$$
I=I_{0} \exp \left(\frac{-\tau(z)}{\sin (\theta)}\right),
$$

dónde $I$ es la irradiancia medida en la superficie e $I_{0}$ es la irradiancia obtenida a partir de la Ecuación (1) y $\theta$ es la altura solar.

Diversos métodos se emplean para medir la Irradiancia de una localidad específica [3-8], y su denominación genérica es la de piranómetro o radiómetros, en ocasiones se usa también la denominación de solarímetros o actinómetros. Según su principio de funcionamiento se clasifica en termoeléctricos y fotoeléctricos, los primeros emplean la conversión de energía radiada en calor y los últimos tienen como fundamento el efecto fotoeléctrico. La radiación que incide sobre un fotodiodo también es capaz de diferenciar el espectro solar por la frecuencia de la onda electromagnética, y mediante el voltaje sobre una unión semiconductora cuantificar la radiación, en ese caso se trata de un piranómetro digital, toda vez que la lectura del voltaje es amplificada y enviada a un convertidor analógico-digital.

El objetivo del presente trabajo es, por una parte, mostrar una metodología para evaluar el espesor óptico atmosférico y la irradiancia solar global. Y por otra parte comunicar la forma de construir un piranómetro digital en forma simple y a muy bajo costo, con diodos LED como elemento optosensor (sección 2). En la sección 3, se reportan las estimaciones de la irradiancia solar global, en la región visible del espectro electromagnético; y el espesor óptico atmosférico para el caso especifico de la ciudad de Valencia (Venezuela), además de la variabilidad horaria y estacional. Las conclusiones se presentan en la última sección.

\section{Materiales y método}

Para estimar el espesor óptico atmosférico en un lugar determinado, debe medirse la irradiancia local promedio (integrada sobre las longitudes de onda del visible) en el lugar considerado, empleando para ello los radiómetros atmosféricos o piranómetros. Primeramente se diseñaron y construyeron piranómetros digitales los cuales cuentan con sub-circuitos que realizan las funciones de captura de datos, procesamiento, y almacenaje, en pantalla, de la irradiancia instantánea (ver Fig. 1).

El procesador analógico-digital, compuesto por un Microchip(C)16F88 funcionando a 1MIPS y una pantalla LCD $16 \times 2$, es el que se encarga de interconectar y procesar las señales provenientes del resto de los sub circuitos mostrados en la Fig. 1. El diseño del radiómetro está basado en un primer prototipo mostrado por Falcón et al [3] construido con un LED rojo, modificado para permitir su digitalización $\mathrm{y}$ mejorando la sensibilidad con la inclusión de un segundo LED azul. Los piranómetros diseñados constan de un amplificador operacional TL074 configurado en modo de convertidor de corriente a voltaje donde se conecta a los LED, usados como elemento optosensor.

Para procesar el voltaje de salida del radiómetro se implementó un PIC-16F88 (Fig 1(c)) el cual posee una entrada análoga digital y una salida a un display LM016L de 16 caracteres por 2 líneas. Cuenta con un único botón para almacenar los datos en pantalla mientras envia los datos al ordenador a través del puerto RS232 (Fig. 1(d)).

En la Fig. 2 se puede apreciar el mecanismo de lectura de ángulo de elevación, allí aparecen imágenes sucesivas de la barra de sensores 


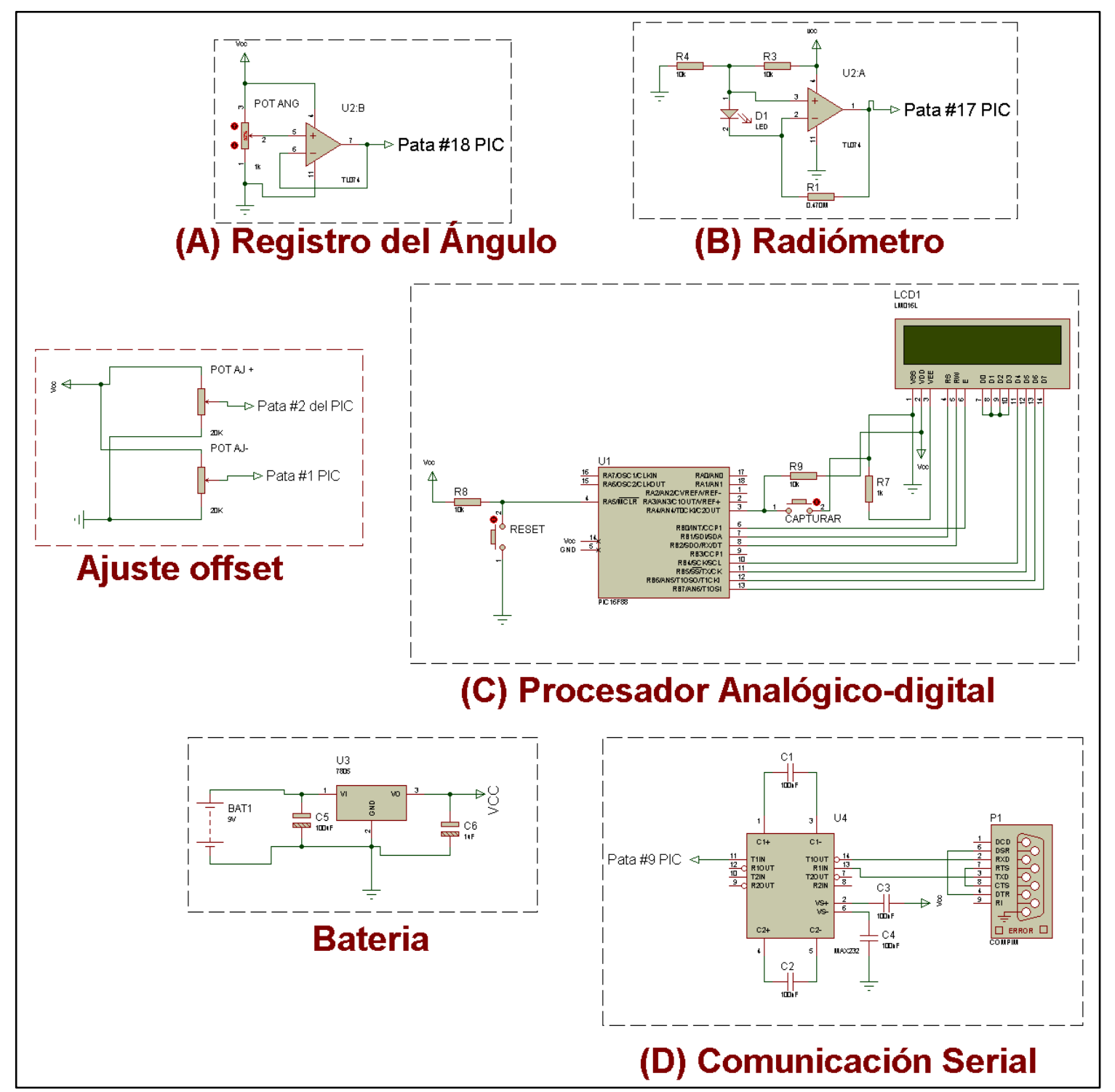

Fig. 1. Esquema eléctrico general del piranómetro digital.

girada de izquierda a derecha, el mismo consta de un potenciómetro en modo divisor de tensión cuya salida de voltaje es proporcional a la posición en la que se encuentre el potenciómetro. En el medio de los dos sensores se coloca una mirilla o apuntador para variar la inclinación de los sensores de acuerdo a la distancia cenital instantánea del sol, de modo que apunten directamente a dicha fuente. Este proceso de colimación y apuntado se efectúa manualmente por un observador entrenado.

Como elementos sensores se utilizaron dos LED de alto brillo: uno rojo con máximo de emisión en $620 \mathrm{~nm}$ y otro azul con máximo en los $472 \mathrm{~nm}$ como se aprecia en la Fig. 3, para obtener el cambio de irradiancia en los dos extremos del espectro visible. Los LEDs son muy eficientes como emisores de luz, pero frecuentemente se soslaya su empleo como fotodetectores.

Para calibrar la salida en voltaje del piranómetro en medidas de irradiancia en $\mathrm{W} / \mathrm{m}^{2}$ se utilizó el integrado TSL-230 Texas Instruments $®$, que posee una salida en frecuencia fácilmente contabilizada por un osciloscopio de $100 \mathrm{MHz}$; para obtener una relación directa entre la frecuencia y la irradiancia instantánea sobre cada uno de los LED, con un error máximo de $1 \mathrm{~W} / \mathrm{m}^{2}$ tanto para el LED rojo y como el azul. 


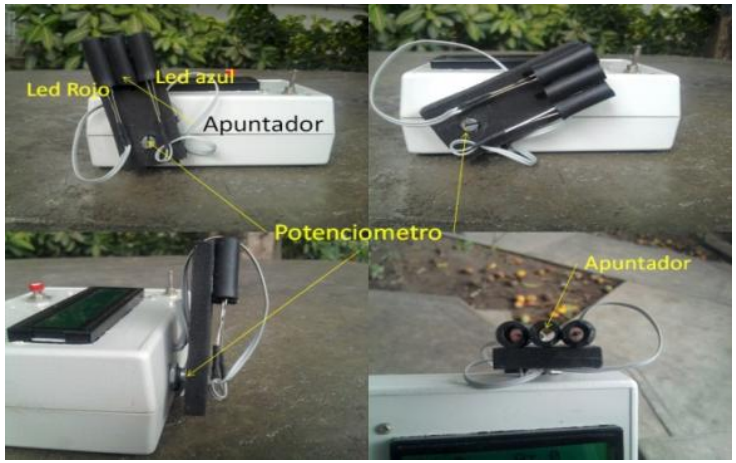

Fig. 2. Conjunto apuntador-colimador-sensores LED.

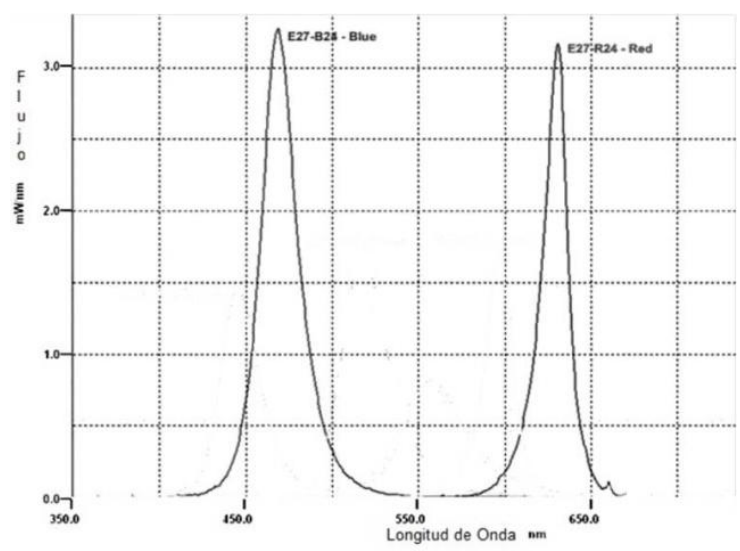

Fig. 3. Curva espectrales de sensibilidad de los LED [9].

El método empleado para determinar la irradiancia instantánea consiste en medir simultáneamente el flujo en las longitudes de onda $620 \mathrm{~nm}$ (rojo) y en los $472 \mathrm{~nm}$ (azul), así como el ángulo de altura solar en función del tiempo, en los instantes en que el disco solar es completamente visible sin nubes interpuestas entre este y el observador. Se emplearon medidas cada hora bajo condiciones de cielos claros y sin nubes. Para ello se posiciona el dispositivo sobre un plano horizontal a 1,5 metros del suelo, orientado de modo que el plano del detector resulte ortogonal a la vertical. La posición se calibra por medio de la mirilla del apuntador-colimador de modo que los rayos solares al incidir sobre el cilindro de la mirilla produzcan una imagen circular de insolación sobre el plano, si la imagen de insolación aparece esferoidal o elíptica se rota el instrumento respecto a la vertical hasta corregirla; si por el contrario aparece como un semicírculo o elipse truncada, se mueve el apuntador en su ángulo de elevación hasta obtener un circulo de insolación de los rayos solares pasando directamente a través del apuntador colimador. Este proceso se efectúa muy rápidamente por un observador entrenado, reduciendo el error del ángulo de elevación hasta en 0,2 grados, verificable por la simulación realizada previamente para una fuente de luz puntual en condiciones de laboratorio.

\section{Resultados y discusión}

La estación de observación para Valencia (Edo Carabobo Venezuela) se ubicó en las coordenadas $10.128611 \mathrm{~N}$ y $68.016111 \mathrm{~W} 481$ msnm, en la sede del laboratorio de Física de la Atmosfera y el Espacio Ultraterrestre de la Universidad de Carabobo. Las medidas de irradiancia se colectaron entre las 7:00 y 17:00 horas locales HLV (HLV=TU-4:30); soslayando los primeros instantes del orto y los últimos instantes del ocaso solar por las limitaciones de visibilidad orográficas. Toda la data se refiere a las observaciones diarias de cielos despejados (cielos claros y sin nubes) a lo largo de un año: entre enero 2012 y enero 2013. Los meses de julio, agosto y septiembre del 2012 no mostraron ningún día con cielos claros que permitieran medir a lo largo de todo el día el disco solar sin interrupciones nubosas en relación al observador, sino solo días con intervalos parcialmente sin nubes, y por ello no se reportan los tales meses. Todos los resultados están referidos a la Hora Legal Venezolana (HLV) que equivale al Tiempo Universal (TU) menos 4:30 horas. Los resultados de las medidas de irradiancia a $620 \mathrm{~nm}$ (LED rojo) y a $472 \mathrm{~nm}$ (LED azul) se resumen en la Fig. 4.

Obsérvese que ambas curvas se ajustan a una variación con tendencia no monótona, y una meseta en las horas próximas a las 9:00 HLV, presumiblemente debida a los procesos convectivos que tienen lugar cuando comienza la evaporación a nivel superficial y la condensación en capas medias de la troposfera al despuntar el alba, y que disminuyen la irradiancia en superficie, que podrían acumular vapor de agua en la atmosfera aun sin la presencia evidente de nubes. Así mismo se observa un decaimiento rápido en las horas posteriores a la 1:30 HLV, 

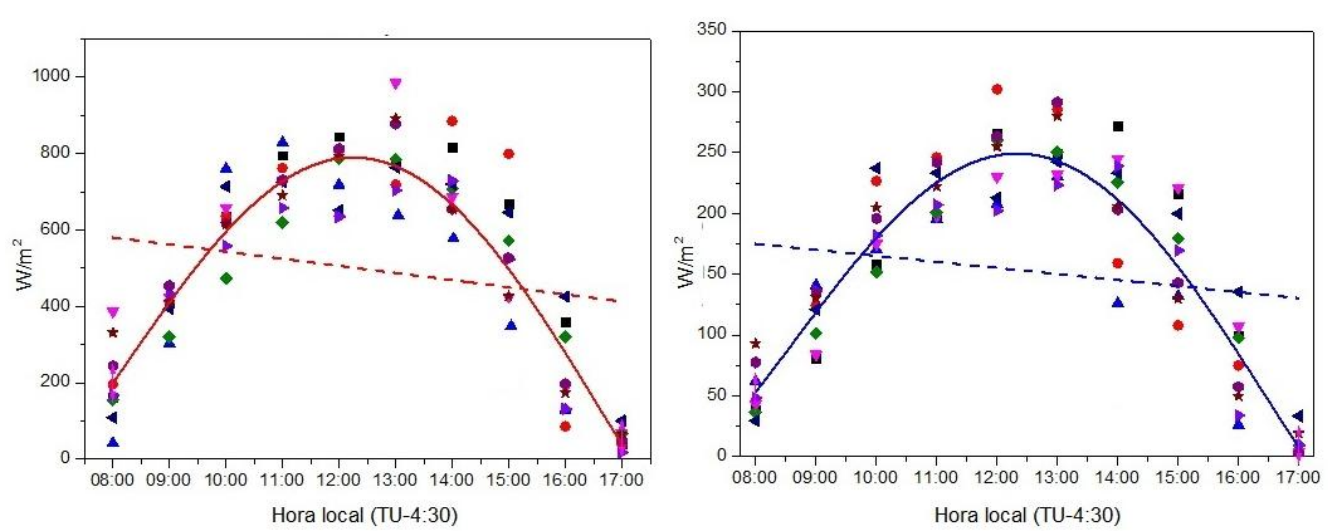

Fig. 4. Variación de la irradiancia en Valencia.
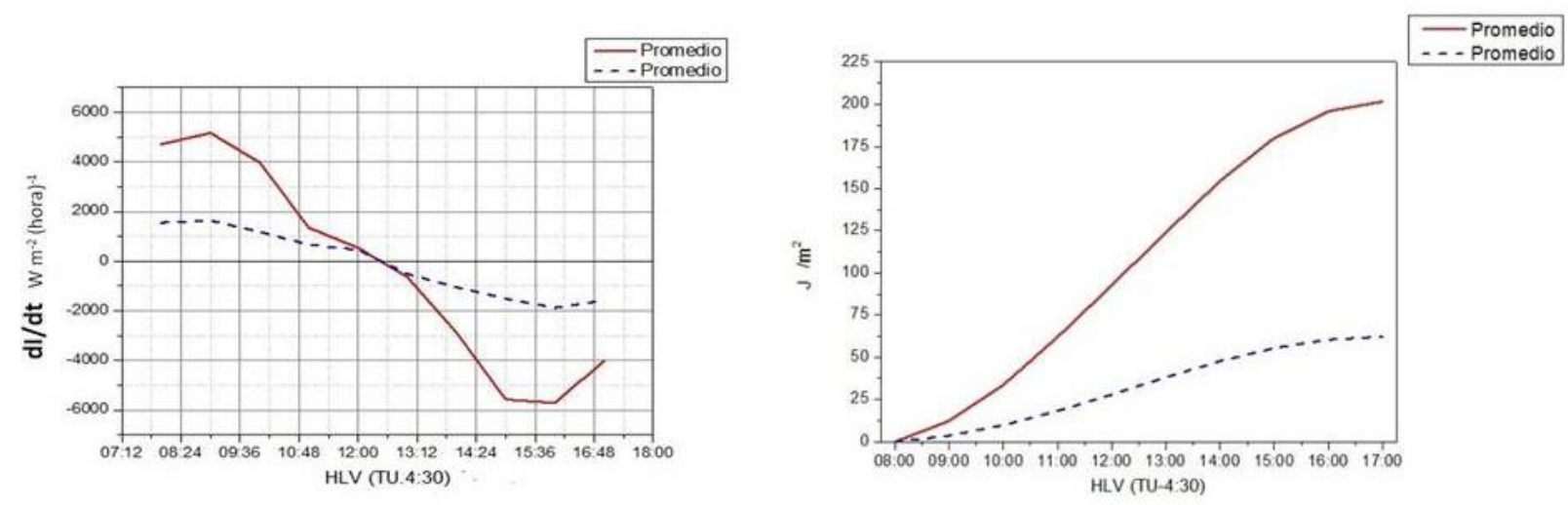

Fig. 5. Variación temporal de la irradiancia horaria (izquierda) y energía radiante promedio acumulada (derecha).

por la formación de nubes en el atardecer, que a pesar de no cubrir el disco solar y permitir las mediciones, son visibles en otras direcciones del cielo.

En la Fig. 5 se resume la derivada y la integral de las irradiancias medias anuales medidas localmente.

La Fig. 5 (izquierda) muestra la ocurrencia del máximo de irradiancia para el LED rojo se produce a las 12:31 HLV con un promedio de $805 \mathrm{~W} / \mathrm{m}^{2}$ y a las $12: 18 \mathrm{HLV}$ con un promedio de $249 \mathrm{~W} / \mathrm{m}^{2}$ para las longitudes de onda mas cortas (LED azul); esta diferencia en la ocurrencia del máximo puede estar asociada a las diferencias de opacidades de las capas troposféricas medias. La Fig. 5 (derecha) muestra la energía radiante promedio acumulada por intervalo horario con un máximo de $200 \mathrm{~J} / \mathrm{m}^{2}$ para los $620 \mathrm{~nm}$ (LED rojo) y 50 $\mathrm{J} / \mathrm{m}^{2}$ para los $472 \mathrm{~nm}$ (LED azul), la diferencia estaría relacionada con la absorbancia selectiva por aerosoles y dispersión de Rayleigh.
Igualmente se observa (Fig. 5 derecha) que la variación horaria del flujo de energía en días soleados y sin nubes, es mucho mayor para las longitudes de onda larga (rojo) que para las longitudes de onda corta (azul).

La Fig. 6 muestra la variación estacional del flujo de energía instantáneo en condiciones de días soleados, con un valor medio de $61 \pm 3 \mathrm{~J} / \mathrm{m}^{2}$

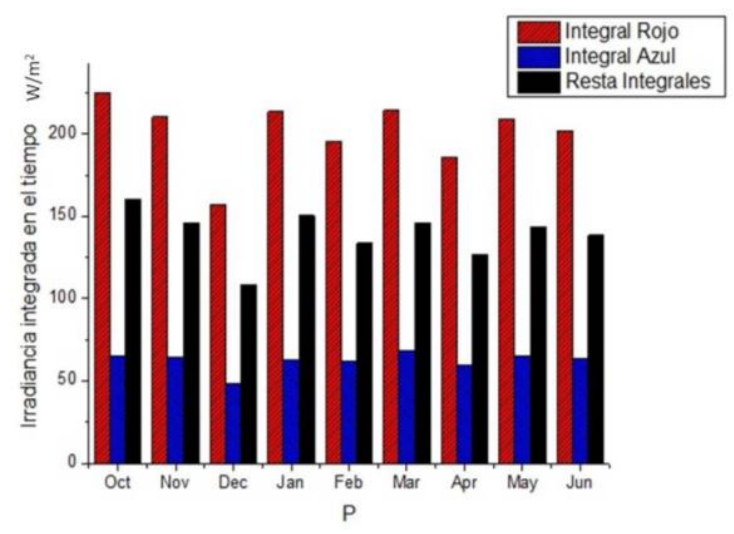

Fig. 6. Variación estacional del flujo de energía instantáneo $\left(\mathrm{J} / \mathrm{m}^{2}\right)$. 

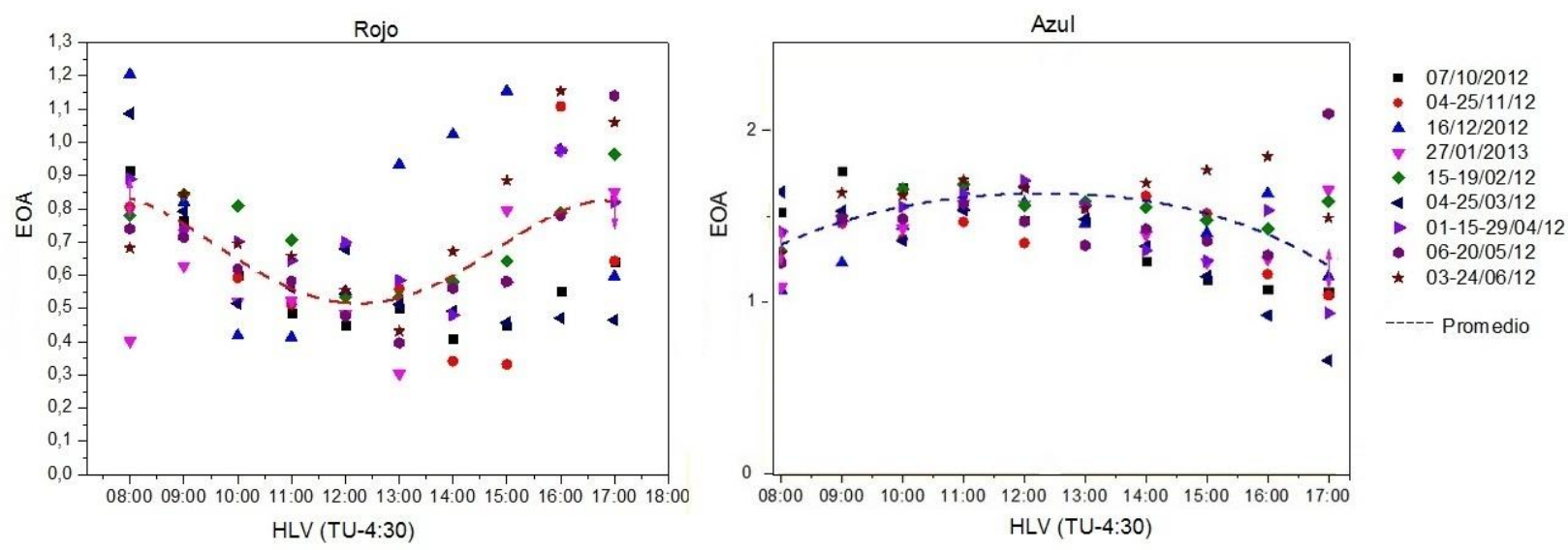

Fig. 7: Variación horaria del coeficiente de espesor óptico atmosférico.

para los $472 \mathrm{~nm}$ (LED Azul) más o menos constante en todo los meses registrados (octubre a Junio) y del orden de $203 \pm 8 \mathrm{~J} / \mathrm{m}^{2}$ para los $620 \mathrm{~nm}$ (LED rojo) con un mínimo en Diciembre, cuando los rayos solares inciden mas oblicuamente para el hemisferio norte, en concordancia con el solsticio de invierno, y se esperaría un máximo en el mes de julio (solsticio de verano) cuando los rayos solares inciden mas perpendicularmente en el hemisferio norte, pero no se cuenta con datos de irradiancia en dicho mes por la abundante nubosidad. Las barras negras muestran que la diferencia del flujo de energía instantáneo entre las longitudes de onda roja y azul es del orden de los $125 \mathrm{~J} / \mathrm{m}^{2}$ para todo el año con un mínimo en diciembre y quizá un máximo a finales de septiembre.

Por otro lado, el espesor óptico atmosférico local y su variabilidad horaria puede ser obtenido a partir de las mediciones de irradiancia empleando la Ec. (2), como se muestran en la Fig. 7.

La Fig. 7 muestra la fuerte dependencia del EOA con la longitud de onda. Para las longitudes de onda cercanas a la región roja del espectro visible, la variación horaria del EOA sigue, grosso modo la atenuación debida al movimiento aparente del Sol, máximo a comienzos y final del día. Para longitudes de onda cercanas al azul la opacidad en cambio muestra la tendencia opuesta, como cabe esperar por cuanto la irradiancia difusa es mayor en las horas cercanas al mediodía (insolación) y es menor a comienzos y final del día. Como se evidencia en las concavidades de la variación horaria del EOA: hacia arriba para el rojo y hacia abajo la correspondiente al azul.

En la Fig. 8(a) se muestran los promedios de EOA en la ciudad de Valencia: los promedios azul y rojo, de manera separada incorporan en diferente cuantía la irradiancia difusa tanto por aerosoles como por la constitución atmosférica (dispersión de Rayleigh. que da el color azul del cielo, y dispersión de Mié debida a los aerosoles). Adicionalmente se muestran los puntos correspondientes a la sustracción entre los datos correspondientes al EOA en azul y en rojo, describiendo una curva cuyo comportamiento es monótono. La interpretación es que la curva continua (Fig. 8 izquierda) representa la diferencia del espesor óptico atmosférico promedio, siendo máximo al amanecer $\mathrm{y}$ atardecer, y mínimo al mediodía como cabria esperar si se considera solamente la irradiancia solar directa.

En la Fig. 8 (derecha) se muestran las derivadas de las tres curvas con detalle de los puntos críticos y las ocurrencias máximas y mínimas. La diferencia de los EOA promedio muestra, como cabe esperar, un mínimo al mediodía (12:02 HLV), en concordancia con el mediodía astronómico verdadero para la longitud de $68.016111^{\circ} \mathrm{W}$ y un uso horario para la longitud de $67,5^{\circ} \mathrm{W}$. No ocurre así con los valores máximos del EOA calculados para las irradiancias en los extremos del espectro, pues el azul se adelanta respecto al mediodía astronómico verdadero y el rojo se retrasa con un máximo a las 12:30 HLV. Es notable el 

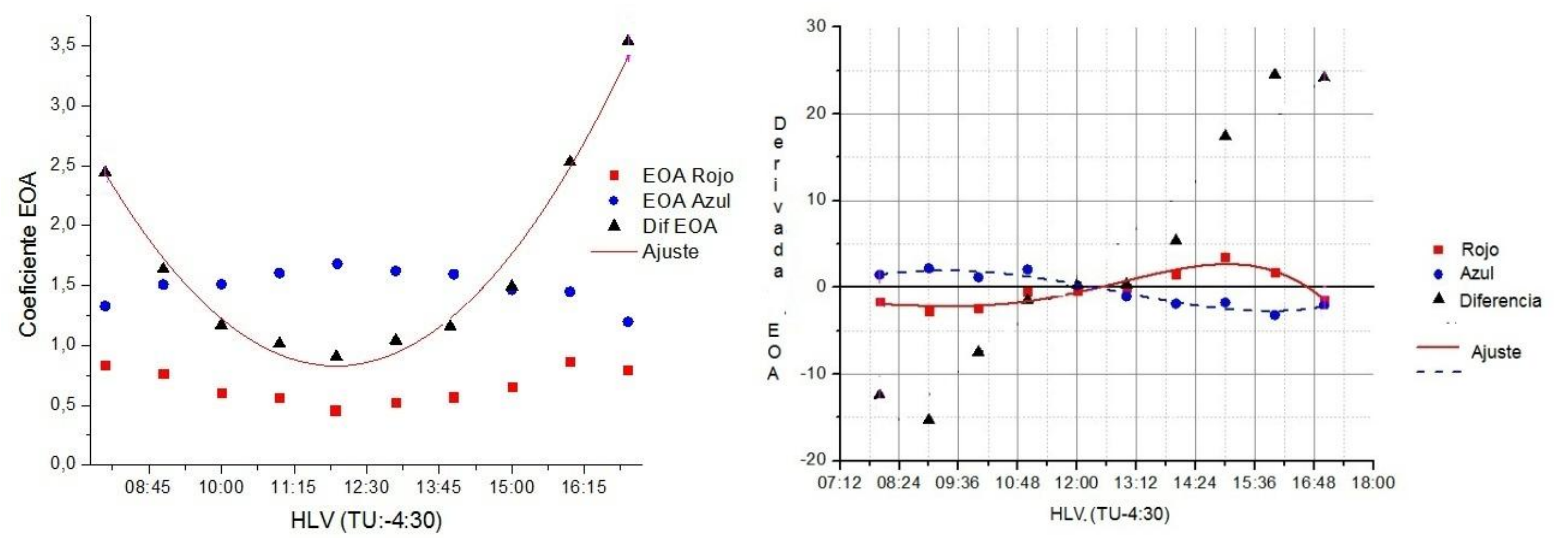

Fig. 8. Variación horaria del EOA promedio local (izquierda) y de su derivada (derecha).

comportamiento monótono de la diferencia de los EOA cuando se compara con las variaciones tomadas en longitudes roja y azul, que presentan un segundo máximo entre las 16:00 y 17:00 HLV. Corroborando la importancia de las mediciones de la irradiancia global en ambos extremos del espectro visible (azul y rojo).

\section{Conclusiones}

El piranometro digital construido empleando diodos LED como elemento foto sensores permite evaluar la irradiancia solar global y su variación horaria, como se mostró en la Fig. 4. Los valores promedio de irradiancia para la ciudad de Valencia (Venezuela) son de 805 $\mathrm{W} / \mathrm{m}^{2}$ para los $620 \mathrm{~nm}$ (LED rojo) y $249 \mathrm{~W} / \mathrm{m}^{2}$ para los $472 \mathrm{~nm}$ (LED azul) con valores máximo de irradiancia a las 12:31 HLV (rojo) y a las 12:18 HLV (azul), como se mostró en las Figs. 4 y 5 . La energía radiante promedio acumulada por intervalo horario varía entre los $200 \mathrm{~J} / \mathrm{m}^{2}$ a la longitud de onda de $620 \mathrm{~nm}$ (rojo) y $50 \mathrm{~J} / \mathrm{m}^{2}$ para los $472 \mathrm{~nm}$ (azul). La diferencia estaría relacionada con la absorbancia selectiva por aerosoles y dispersión de Rayleigh. Por otro lado la Fig. 6 mostró que la variación estacional del flujo de energía instantáneo de la irradiancia solar global en la ciudad de Valencia (Venezuela), es del orden de $70 \mathrm{~J} / \mathrm{m}^{2}$ para los $472 \mathrm{~nm}$ (azul) y del orden de $175 \mathrm{~J} / \mathrm{m}^{2}$ para los $620 \mathrm{~nm}$ (rojo). Y sus fluctuaciones presentarían un mínimo en diciembre y máximo en Septiembre, en concordancia con los solsticios.
En las Figs. 7 y 8 también se pudo observar que los datos del EOA para el sensor rojo son cóncavas hacia arriba, mientras que para el azul son cóncavas hacia abajo, ello arroja información sobre cómo es la dispersión en la atmosfera para ambas longitudes de onda, en acuerdo con la teoría de dispersión de Mie. En la Fig. 8 la curva de ajuste del EOA considerando la diferencia de irradiancias instantáneas (medidas por el sensor rojo menos las medidas por el sensor azul) muestra la contribución neta de irradiancia directa. Ello es debido a que la irradiancia en el espectro del azul es predominantemente difusa, y la contribución en la banda roja es la combinación de las componentes directa $y$ difusa. Al sustraer ambas bandas resulta evidente el resultado. Se destaca la importancia de realizar medidas continuas a lo largo del año y de la incorporación de medidas en los extremos del espectro visible, cuya sustracción permite identificar la variación de la irradiacia solar directa y cuantificar la componente difusa en la insolación diaria.

\section{Agradecimientos}

Este trabajo ha sido financiado por el Fondo Nacional de Ciencia Y Tecnología (FONACIT) de la Republica Bolivariana de Venezuela, bajo el $\mathrm{N}^{\circ}$ 2011-000326 del Proyecto Estratégico “Caracterización de Fenómenos Transitorios en la Troposfera Baja: Electrometeoros, Litometeoros, Microtornados y Trombas Marinas". 